\title{
A finite-difference lattice Boltzmann approach for gas microflows
}

\author{
G. P. Ghiroldi and L. Gibelli* \\ Dipartimento di Scienze e Tecnologie Aerospaziali, Politecnico di Milano, 20156 Milano, Italy
}

(Dated: September 21, 2018)

\begin{abstract}
Finite-difference Lattice Boltzmann (LB) models are proposed for simulating gas flows in devices with microscale geometries. The models employ the roots of half-range Gauss-Hermite polynomials as discrete velocities. Unlike the standard LB velocity-space discretizations based on the roots of full-range Hermite polynomials, using the nodes of a quadrature defined in the half-space permits a consistent treatment of kinetic boundary conditions. The possibilities of the proposed LB models are illustrated by studying the one-dimensional Couette flow and the two-dimensional driven cavity flow. Numerical and analytical results show an improved accuracy in finite Knudsen flows as compared with standard LB models.
\end{abstract}

PACS numbers: 47.11.-j, 47.15.-x, 47.61.-k

The outgrowing development of microelectromechanical systems (MEMS) has spurred interest in studying gas flows in devices with microscale geometries. These flows, which are referred to as gas microflows, are usually distinguished by relatively large Knudsen numbers and small Mach numbers. Because of the large Knudsen numbers, the conventional hydrodynamic approach breaks down and a description based on the Boltzmann equation is required. The low Mach numbers permit to replace the collision integral of the Boltzmann equation with a simpler kinetic model, such as the one proposed by Bathnagar, Gross and Krook and, independently, by Welander (BGKW), as well as to linearize the resulting kinetic equation around the equilibrium state [1]. Numerous studies on gas microflows based on the numerical solution of the linearized BGKW kinetic model equation [2-4] and/or the linearized Boltzmann equation [5] have been reported over the years. Recently, the Lattice Boltzmann (LB) method has attracted considerable interest as an alternative tool for studying gas flows in microfluidic devices $[6-12]$. Although it was evolved from lattice-gas cellular automaton models for mimicking the Navier-Stokes hydrodynamics, the LB method can potentially describe gas microflows since it can be viewed as the discrete ordinate method to solve the linearized BGKW kinetic model equation [13, 14]. By using the same notation as in Ref. [1], the non dimensional form of LB models in the absence of external force fields reads

$$
\frac{\partial f_{\alpha}}{\partial t}+\boldsymbol{\xi}_{\alpha} \cdot \nabla f_{\alpha}=-\frac{1}{\mathrm{Kn}}\left(f_{\alpha}-f_{\alpha}^{e q}\right),
$$

where the Knudsen number is defined as $\mathrm{Kn}=$ $\mu_{0} \sqrt{R T_{0}} /\left(p_{0} l\right)$ with $\mu_{0}, T_{0}, p_{0}$ the reference gas viscosity, temperature and pressure, respectively, $l$ the characteristic length and

$$
\begin{array}{r}
f_{\alpha}(\boldsymbol{r}, t)=w_{\alpha} f\left(\boldsymbol{r}, \boldsymbol{\xi}_{\alpha}, t\right) / \omega\left(\boldsymbol{\xi}_{\alpha}\right), \\
f_{\alpha}^{e q}(\boldsymbol{r}, t)=w_{\alpha} f^{e q}\left(\boldsymbol{r}, \boldsymbol{\xi}_{\alpha}, t\right) / \omega\left(\boldsymbol{\xi}_{\alpha}\right),
\end{array}
$$

being

$$
\omega(\boldsymbol{\xi})=\frac{1}{(2 \pi)^{d / 2}} \exp \left(-\frac{\xi^{2}}{2}\right) .
$$

In Eqs. (2) and (3), $f$ is the distribution function, $f^{e q}$ is the $d$-dimensional equilibrium Maxwellian and $w_{\alpha}, \boldsymbol{\xi}_{\alpha}$ are the $n$ weights and nodes determined from a quadrature formula, respectively [15]. Since we are considering isothermal flows, the terms related to the temperature in the second-order approximation of the equilibrium Maxwellian can be disregarded and $f^{e q}$ can thus be written as

$$
f^{e q}(\boldsymbol{r}, t)=\omega(\boldsymbol{\xi}) \rho(\boldsymbol{r}, t)[1+\boldsymbol{\xi} \cdot \boldsymbol{u}(\boldsymbol{r}, t)],
$$

where $\rho$ and $\boldsymbol{u}$ are the density and macroscopic velocity, respectively, which can be computed by sums over the discrete velocity set

$$
\begin{aligned}
\rho(\boldsymbol{r}, t) & =\sum_{\alpha=1}^{n} f_{\alpha}(\boldsymbol{r}, t), \\
\rho(\boldsymbol{r}, t) \boldsymbol{u}(\boldsymbol{r}, t) & =\sum_{\alpha=1}^{n} \boldsymbol{\xi}_{\alpha} f_{\alpha}(\boldsymbol{r}, t) .
\end{aligned}
$$

Likewise, boundary conditions can be derived by a direct discretization of Maxwell's diffuse-specular scattering kernel [16]. For the sake of simplicity, let us consider a plane wall at $y=0$ with reference temperature and constant velocity $u_{w}$ in the positive $x$-direction. Let us further suppose that the gas fills the half space $y>0$ and molecules which strike the wall are re-emitted according to Maxwell's scattering kernel with complete accommodation. The discrete form of the kinetic boundary condition at a point $\boldsymbol{r}_{w}$ of the solid surfaces reads

$$
f_{\alpha}\left(\boldsymbol{r}_{w}, t\right)=\omega\left(\boldsymbol{\xi}_{\alpha}\right) \rho_{w}\left(\boldsymbol{r}_{w}, t\right)\left(1+\xi_{x, \alpha} u_{w}\right), \quad \xi_{y, \alpha}>0 .
$$

In Eq. (8), $\rho_{w}$ can be obtained through the impermeability condition, which states that the normal component of the gas velocity on the wall vanishes 
TABLE I: Nodes, $\xi_{\alpha}$, and weights, $w_{\alpha}$, of the one-dimensional half-range Gauss-Hermite quadrature.

\begin{tabular}{ccc}
\hline \hline Quadrature & $\xi_{\alpha}$ & $w_{\alpha}$ \\
\hline$D_{1} Q_{4}^{h}$ & $\mp 0.4245383286$ & 0.3613798911 \\
& $\mp 1.77119083$ & 0.1386201089 \\
$D_{1} Q_{6}^{h}$ & $\mp 0.2694842630$ & 0.2516453504 \\
& $\mp 1.199609295$ & 0.2236832664 \\
$D_{1} Q_{8}^{h}$ & $\mp 2.545268446$ & 0.0246713831 \\
& $\mp 0.1891884657$ & 0.1835325640 \\
& $\mp 0.8829284442$ & 0.2375842404 \\
& $\mp 1.898635201$ & 0.07528686870 \\
& $\mp 3.199890790$ & 0.003596326913 \\
\hline \hline
\end{tabular}

$$
\rho_{w}\left(\boldsymbol{r}_{w}, t\right)=-(2 \pi)^{1 / 2} \sum_{\xi_{y, \alpha}<0} \xi_{y, \alpha} f_{\alpha}\left(\boldsymbol{r}_{w}, t\right) .
$$

For two-dimensional flows and very low Knudsen numbers, the $D_{2} Q_{9}$ model provides accurate results. We here use the standard terminology and denote by $D_{m} Q_{n}$ the $m$ dimensional LB models with $n$ discrete velocities. As Kn increases, high-order LB models are needed to correctly reproduce non equilibrium effects, such as the velocity slip at the solid walls and the nonlinear stress-strain relationship within the Knudsen layer [9]. In the framework of single relaxation time modeling, several high-order LB models have been developed. Some have been derived by using a local mean free path in order to account for the presence of solid surfaces [6]. Although these models have been shown to be effective in many applications, they are phenomenological in nature and, as such, not perfectly general. Composite models have also been developed which result from the superposition of discrete velocities determined from odd and even quadrature formula [7]. However, the most common strategy is using a greater number of discrete velocities determined from a full-range Gauss-Hermite quadrature [9, 10], possibly adopting a multiscale approach to contain the increase in the computational cost [8]. However, more discrete velocities do not guarantee an improved accuracy [9 11]. It has been demonstrated that this is due to the quadrature effect in dealing with the boundary conditions [12]. As a matter of fact, abscissae of the full-range Gauss-Hermite quadrature schemes are derived to obtain accurate evaluation of the moments of the distribution function defined over the entire velocity space. In contrast, they provide only an approximately estimate of the half-range integrals that enter in the formulation of kinetic boundary conditions [16].

In this Rapid Communication, we want to show that non-equilibrium gas flows can be more accurately described by using a discrete velocity set different from the one employed by standard finite-difference LB models. More specifically, we propose finite-difference LB models which use the roots of half-range Hermite polynomials as quadrature nodes. Table $\llbracket$ gives nodes and weights of the one-dimensional half-range Gauss-Hermite quadrature. The two-dimensional quadrature can be obtained from the tensor product of the corresponding one-dimensional quadrature. Notice should be made that the roots of halfrange Hermite polynomials are irrational and therefore the resulting fully-discrete numerical schemes are computationally more demanding than the simpler "streamand-collide" algorithm. The exact space discretization of the advection step of on-lattice LB models is therefore no longer possible and the potential high efficiency of their parallel implementations is partially lost. However, off-lattice schemes may offer some advantages such as enhanced geometrical flexibility and, as shown in the present work, the capability of describing rarefaction effects. The use of high performance computing is still feasible but the porting of algorithms involving finitedifference approximations certainly requires some additional effort [17]. In comparison with alternative finitedifference LB models, the proposed velocity space discretization permits to explicitly account for the discontinuity of the distribution function and therefore leads to a faster convergence of the solution close to solid surfaces. In kinetic theory applications, the importance of a consistent treatment of boundary conditions has been recognized as early as the sixties of the past century [18] and the half-range discrete ordinate method has been widely used since then [19 23]. By contrast, in LB simulations of gas flows in microchannels, most of high-order LB models use the roots of full-range Hermite polynomials as discrete velocities [6 -8]. Although it is well known that finite-difference LB models can be developed from different quadrature formula [15], to the authors' knowledge, no previous works have pointed out that the quadrature based on half-range Hermite polynomials can easily address the issue of boundary conditions for the LB simulations of gas microflows. An important correspondence can be identified between the approach developed in the present work and the moment method presented in Ref. 21] for studying gas microflows. There, the isothermal linearized BGKW kinetic model equation has been solved by expanding the distribution function as a series of half-range Hermite polynomials. Expansion coefficients are the moments of the distribution function which, in turn, are strictly related to the macroscopic quantities. By using the half-range Gauss-Hermite quadrature formula for evaluating these integrals, a one-to-one correspondence can be identified between the expansion coefficients and the values of the distribution function at the roots of half-range Hermite polynomials. The two approaches are therefore equivalent even though, from the computational standpoint, the formulation which is here proposed is more efficient. It is worth noticing that already in Ref. [16] it has been pointed out that the accuracy in dealing with boundary conditions can be improved by evaluating integrals which enter in their definition by means of a quadrature formula defined in the half-space. However, this possibility has not been further developed because of the mismatch be- 


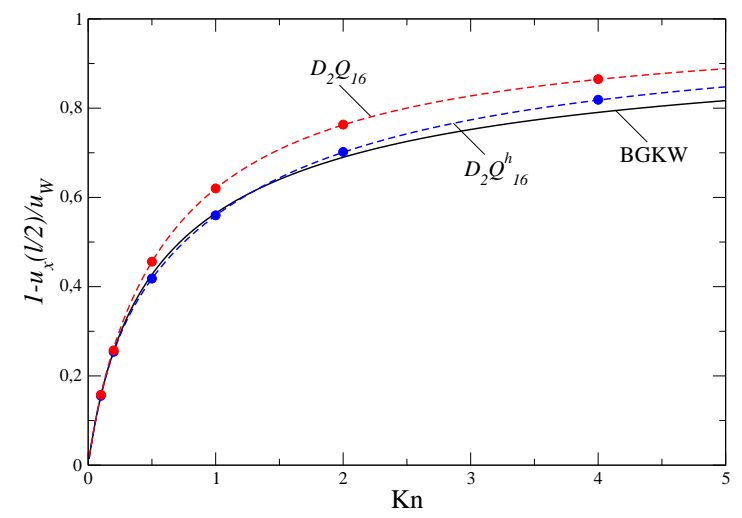

FIG. 1: Velocity slip at the upper plate versus the Knudsen number.

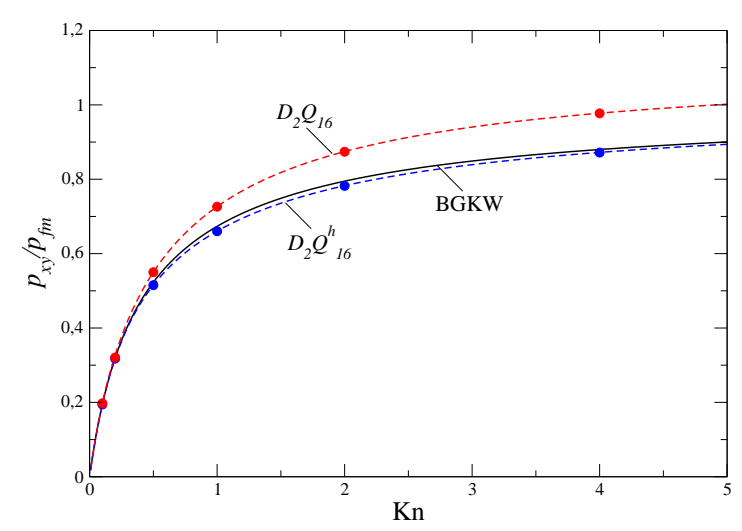

FIG. 2: Reduced $x y$-component of the stress tensor versus the Knudsen number.

tween the nodes of the quadrature used at the boundary and those in the bulk. In the present work, however, the nodes of half-range Gauss-Hermite quadrature formula are used in the whole domain. We demonstrate, both analytically and numerically, that the proposed approach can be used to simulate gas microflows by studying two classical driven boundary value problems, i.e., the Couette and the cavity flows. Both problems are regarded as two-dimensional and the driven velocity is assumed sufficiently low so that the gas flow can be considered in the linearized regime [24]. A fully discrete numerical scheme is derived by a first-order time splitting of the evolution operator and couples a first-order upwind scheme for the transport step with a first-order explicit Euler scheme for the relaxation step. More sophisticated methods should be used for both coupling and solving transport and relaxation steps if a more accurate and efficient numerical scheme is needed. However, the main aim of the work is to show that an improved accuracy in describing finite Knudsen flows can be achieved by using the roots of half-range Hermite polynomials as discrete velocities. This has been assessed by running the same numerical code twice, once with nodes and weights as given by full-range Gauss-Hermite quadrature and once with nodes and weights as given by half-range GaussHermite quadrature. The validity of this comparison is therefore independent on the numerical scheme that has been used to solve the discrete kinetic equation.

In the Couette flow problem, the upper plate moves in the $x$ direction with a velocity $u_{w}$ and the lower plate moves with a velocity $-u_{w}$. Diffuse boundary conditions have been implemented at the plates and periodic boundary conditions have been utilized at opposite ends of the channels. The two plates are separated by the distance $l$. It is worth noticing that the proposed models employ the quadrature nodes of the positive and negative half-range Gauss-Hermite quadrature formula as discrete velocities both in the $x$ - and $y$-directions. However numerical computations showed that the same results are obtained if the $x$-direction of the velocity space is discretized by using the roots of full-range Hermite polynomials. By proceeding as in Ref. 21], it is not difficult to obtain a closedform solution of the $D_{2} Q_{16}^{h}$ LB model for the stationary planar Couette flow

$$
\begin{aligned}
u_{x}(y / l) & =u_{w} \frac{\left[\sinh \left(\frac{0.9494}{K n}\right)+0.7978 \cosh \left(\frac{0.9404}{K n}\right)\right] \frac{y}{l}+0.5642 \mathrm{Kn} \sinh \left(\frac{1.881}{\mathrm{Kn}} \frac{y}{l}\right)}{(0.7071 \mathrm{Kn}+0.3989) \cosh \left(\frac{0.9404}{\mathrm{Kn}}\right)+(1.128 \mathrm{Kn}+0.5) \sinh \left(\frac{0.9404}{\mathrm{Kn}}\right)} \\
\frac{\sqrt{R T_{0}} p_{x y}}{u_{w} p_{0}} & =-\mathrm{Kn} \frac{\sinh \left(\frac{0.9494}{\mathrm{Kn}}\right)+0.7979 \cosh \left(\frac{0.9404}{\mathrm{Kn}}\right)}{(0.7071 \mathrm{Kn}+0.3989) \cosh \left(\frac{0.9404}{\mathrm{Kn}}\right)+(1.128 \mathrm{Kn}+0.5) \sinh \left(\frac{0.9404}{\mathrm{Kn}}\right)} .
\end{aligned}
$$

Beside their intrinsic interest, Equations (10) and (11), as well as a similar closed-form solution obtained in Ref. 25] for the $D_{2} Q_{16}$ model, are of practical importance in that they permit to validate the numerical code. Figures 1 and 2] show the velocity slip, $1-u_{x} / u_{w}$, at the upper plate and the ratio between the $x y$-component of the stress tensor, $p_{x y}$, and the value of the pressure in the free molecular regime, $p_{f m}=-2 p_{0} u_{w} / \sqrt{\pi R T_{0}}$, versus the Knudsen 

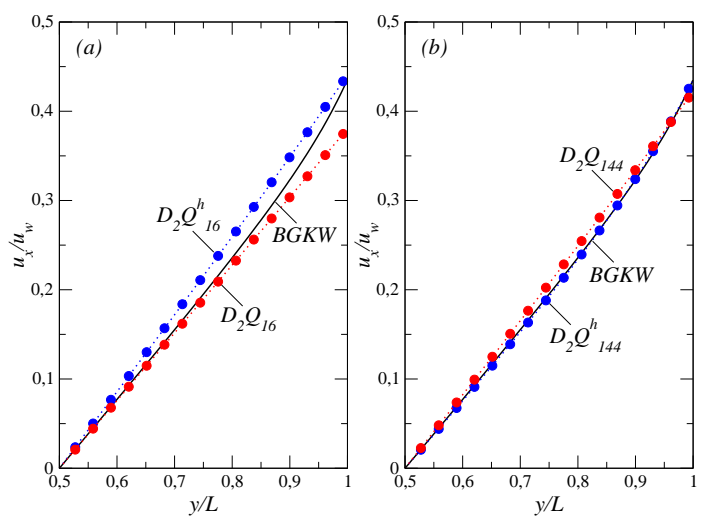

FIG. 3: Nondimensional velocity profile through the halfchannel. $\mathrm{Kn}=1$. (a) $n=16$, (b) $n=144$.

number, Kn, respectively. Both Figures use the same conventions. Solid line are the solutions of the linearized BGKW kinetic model equation obtained by means of the moment method described in Ref. 21]. Dashed lines are the closed-form solutions of the $D_{2} Q_{16}^{h}$ model, as given by Eqs. (10) and (11) evaluated at $y / l=1 / 2$, and of the $D_{2} Q_{16}$ model, which is reported in Ref. [25]. Solid circles are the numerical solutions of the $D_{2} Q_{16}^{h}$ and $D_{2} Q_{16}$ models. Only even-order LB models have been considered since they perform significantly better than those with an odd-order quadrature no matter how the order is [9, 10]. As it is clearly shown, although the same number of discrete velocities is employed, the $D_{2} Q_{16}^{h}$ model gives much improved results as compared with standard $D_{2} Q_{16}$ model. The prediction of the velocity slip shows a very good match with the results obtained by solving the linearized BGKW kinetic model equation not only in the continuum and slip flow regimes but also in the early transition regime. The $x y$-component of the stress tensor shows a quite good agreement even in a wider range of Knudsen numbers. Convergence of the results at $\mathrm{Kn}=1$ with increasing number of discrete velocities is reported in Fig. 3 for the velocity profile. Solid line is the solution of the linearized BGKW kinetic model equation obtained by means of the moment method described in Ref. 21]. Dotted lines with solid circles are the numerical solutions obtained by using $n=16$ (left panel) and $n=144$ (right panel) discrete velocities, respectively. The $D_{2} Q_{16}$ model provides a slightly better description of the gas behavior in the bulk of the flow. A possible explanation is that the full-range Gauss-Hermite quadrature with $n=16$ discrete velocities integrates exactly continuous polynomials of order five in each velocity component whereas the halfrange Gauss-Hermite quadrature with the same number of nodes integrates exactly linear polynomials with a possible discontinuity at the origin. It is thus reasonable that the former can be more accurate in the bulk of the flow where the distribution function is continuous whereas the latter can perform better close to the solid surface where the distribution function is expected to be discontinuous.
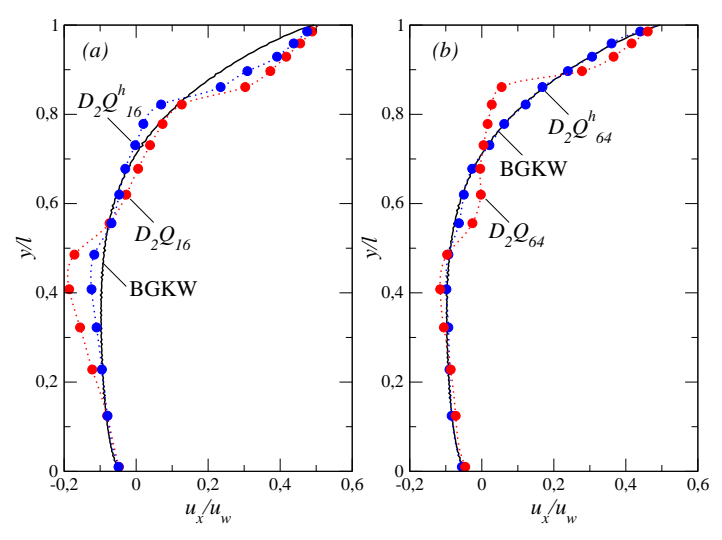

FIG. 4: Profile of the nondimensional horizontal component of the mean velocity crossing the center of the cavity. $\mathrm{Kn}=0.05$. (a) $n=16$, (b) $n=64$.

Nevertheless, as results with $n=144$ clearly show, using the roots of half-range Hermite polynomials as discrete velocities greatly speed-up the convergence to the kinetic theory solution. A similar analysis for the $x y$-component of the stress tensor shows that the numerical prediction of the $D_{2} Q_{16}^{h}$ model at $\mathrm{Kn}=1$ is affected by a relative error which is lower than $5 \%$ and at least 12 nodes per each velocity component should be used for the standard high-order LB models to achieve the same level of accuracy. The possibilities of the proposed models are further illustrated by solving the square driven cavity flow problem [26]. All the walls have a length $l$ and are fixed and isothermal. The flow is driven by the uniform translation of the top. Numerical simulations have been carried out for Knudsen numbers in the range [0.05, 0.4]. This problem constitutes a severe test to assess the capability of the proposed velocity space discretization to describe the gas behavior in complex geometries. Indeed, the discontinuities which exist at the four corners, particularly the two at the top, propagate inside the computational domain and might cause the quadrature to fail. Since the discontinuities decay with distance owing to molecular collisions, it is expected that these numerical problems become more severe as the Knudsen number is increased. In Fig. 4, the profile of the nondimensional horizontal component of the macroscopic velocity, $u_{x} / u_{w}$, crossing the center of the cavity are thus show for the higher Knudsen number we considered, $\mathrm{Kn}=0.4$. Solid line is the solution of the linearized BGKW equation obtained with the numerical method described in Ref. [26]. Dotted lines with solid circles are the numerical solutions provided by full- and half-range LB models with $n=16$ (left panel) and $n=64$ (right panel) discrete velocities. As it was clearly shown, both methods suffer from an unphysical oscillatory behavior due to the difficulty of the quadratures to evaluate a region where discontinuities are present. Although more sophisticated methods can be adopted [26], we here simply notice that the problem 
can be overcome by a reasonable increase in the number of discrete velocities. As for the case of the Couette flow, the proposed approach shows better convergence properties. For instance, the errors in the $\mathcal{L}^{1}$-norm of fulland half-range LB models using $n=16$ discrete velocities are 0.0445 and 0.0280 , respectively, and reduce to 0.0218 and 0.00354 for $n=64$. Table $\amalg$ reports a comparison of the values of the mean dimensionless shear stress along the moving plate, $D$, and the dimensionless flow rate of the main vortex, $G$, obtained by the different models [26]. The predictions of the $D_{2} Q_{16}^{h}$ model show a better agreement with the kinetic theory results than those of the $D_{2} Q_{16}$ model. In spite of the unphysical oscillations which affect the macroscopic velocity, the drag coefficient converges up to two significant figures. Instead the error in the reduced flow rate is less than $1 \%$ for $\mathrm{Kn}=0.05$ but rapidly increases for greater Knuden numbers. However, as shown in Ref. [21], a quite good agreement can be found up to $\mathrm{Kn}=10$ if the $D_{2} Q_{36}^{h}$ is used.

TABLE II: Drag coefficient, $D$, and reduced flow rate, $G$, versus the Knudsen number, Kn.

\begin{tabular}{cccccccc}
\hline \hline & \multicolumn{3}{c}{$D$} & & \multicolumn{3}{c}{$G$} \\
\cline { 2 - 3 } \cline { 6 - 8 } Kn & BGKW & $D_{2} Q_{16}^{h}$ & $D_{2} Q_{16}$ & & BGKW & $D_{2} Q_{16}^{h}$ & $D_{2} Q_{16}$ \\
\hline 0.05 & 0.258 & 0.256 & 0.270 & & 0.154 & 0.153 & 0.162 \\
0.1 & 0.328 & 0.327 & 0.350 & & 0.136 & 0.128 & 0.153 \\
0.2 & 0.425 & 0.417 & 0.467 & & 0.121 & 0.109 & 0.148 \\
0.4 & 0.385 & 0.384 & 0.421 & & 0.110 & 0.0961 & 0.145 \\
\hline \hline
\end{tabular}

To summarize, we have shown that, in comparison with full-range finite-difference high-order LB models, using the nodes of half-range Gauss-Hermite quadrature as discrete velocities permits to consistently deal with kinetic boundary conditions and thus to achieve a more accurate description of the gas behavior close to solid surfaces. Applications to one- and two-dimensional driven boundary value problems show that, even using a small number of discrete velocities, accurate results can be obtained in a wide range of Knudsen numbers which extends up to the early transition regime. The proposed approach is also of interest in that, as it can be deduced from Ref. [21], half-range LB models with $N$ discrete velocities simplify to full-range LB models with $N / 2^{d}$ velocities far away from solid surfaces, being $d$ the dimension of the physical space. This suggest the development of a hybrid approach based on their coupling which combines the capability of half-range LB models to accurately describe the gas behavior in the Knudsen layers with the higher computational efficiency of full-range LB models when applied to the bulk of the gas.

\section{Acknowledgments}

The authors would like to thank Prof. A. Frezzotti for critically reading the paper and Prof. D. Valougeorgis for providing his numerical code. This work has been partly supported by "Progetto Giovani Ricercatori GNFM 2013", Regione Lombardia and CILEA Consortium through a LISA Initiative (Laboratory for Interdisciplinary Advanced Simulation) 2011 grant [link:http://lisa.cilea.it].
[1] C. Cercignani, The Boltzmann Equation and Its Applications, Springer-Verlag, New York, 1988.

[2] S. Lorenzani, L. Gibelli, A. , A. Frangi, and C. Cercignani, Nano. Micro. Therm. Eng. 11 (1-2), 211 (2007).

[3] S. Naris and D. Valougeorgis, Phys. Fluids 19, 067103 (2007).

[4] A. Alexeenka, S. Chigullapalli, J. Zeng, X. Guo, A. Kovacs, and D. Peroulis, Reliab. Eng. Syst. Safe. 96, 11711183 (2011).

[5] C. Cercignani, A. Frangi, A. Frezzotti, G. P. Ghiroldi, L. Gibelli, and S. Lorenzani, Sens. Lett. 6 (1), 121-129 (2008).

[6] Y. H. Zhang, X. J. Gu, R. W. Barber, and D. R. Emerson, Phys. Rev. E. 74, 046704-7 (2006).

[7] L. de Izarra, J. L. Rouet, and B. Izrar, Phys. Rev. E 84, 066705 (2011).

[8] J. Meng, Y. Zhang, and X. Shan, Phys. Rev. E 83, 046701 (2011).

[9] S. H. Kim, H. Pitsch, and I. D. Boyd, J. Comput. Phys. 227, 8655-8671 (2008).

[10] J. Meng and Y. Zhang, J. Comput. Phys. 230, 835-849 (2011).

[11] J. Meng and Y. Zhang, Phys. Rev. E 83, 036704 (2011).

[12] Y. Shi, P. L. Brookes, Y. W. Yap, and J. E. Sader, Phys.
Rev. E 83, 045701(R) (2011).

[13] X. He and L. S. Luo, Phys. Rev. E 56 (6), 6811-6817 (1997).

[14] X. Shan and X. He, Phys. Re. Lett. 80 (1), 65-68 (1998).

[15] X. Shan, X. Feng, and H. Chen, J. Fluid. Mech. 550, 413-441 (2006).

[16] S. Ansumali and I. V. Karlin, Phys. Rev. E. 66, 026311 (2002).

[17] A. Frezzotti, G.P. Ghiroldi, and L. Gibelli, Comput. Fluids 50, 136-146 (2011).

[18] E. P. Gross, E. A. Jackson, and S. Ziering, Ann. Phys. 1, 141-167 (1957).

[19] A. B. Huang and D. P. Giddens, 5th Int. Symp. on Rarefied Gas Dynamics, 481-504 (1967).

[20] L. B. Barichello, M. Camargo, P. Rodrigues, and C. E. Siewert, ZAMP 52, 517-534 (2001).

[21] A. Frezzotti, L. Gibelli, and B. Franzelli, Cont. Mech. Ther. 21 (6), 495-509 (2009).

[22] L. Gibelli, Phys. Fluids 24, 022001 (2012).

[23] G. P. Ghiroldi and L. Gibelli, arXiv:1303.6825 1-23 (2013).

[24] G. P. Ghiroldi, L. Gibelli, P. Dagna, A. Invernizzi, 28th International Symposium on Rarefied Gas Dynamics, 913 July 2012, Zaragoza, Spain, AIP Conference Proceed- 
ings American Institute of Physics, 1501, pp. 735-741 (2012).

[25] S. Ansumali, I. V. Karlin, S. Arcidiacono, A. Abbas, and N. I. Prasianakis, Phys. Rev. Lett. 98, 124502 (2007).
[26] S. Naris and D. Valougeorgis, Phys. Fluids 17, 097106 (2005). 